\title{
DIFFERENTIAL GEOMETRY OF AN $m$-DIMENSIONAL MANIFOLD IN A EUCLIDEAN SPACE OF $n$ DIMENSIONS*
}

BY

\author{
CHARLES E. WILDER
}

\section{INTRODUCTION}

This paper had its inspiration in the Einstein Theory. It grew out of an attempt to give geometric interpretation to the Einstein equations for material free space. It was first developed by the methods of Vosst, and Beezł, but later changed to the simpler notation of Wilson and Moore§, which is a combination of vector analysis with the absolute calculus of Ricci and LeviCivita||. In Voss is to be found the differential geometry of varieties of $n-1$ dimensions in a space of $n$ dimensions. In the article of Wilson and Moore is developed the geometry of a two-dimensional manifold in $n$ dimensions. These are the two natural extensions of surface theory to a higher number of dimensions. The object of this paper is to begin the study of the intermediate cases.

The method of the absolute calculus is developed in great detail in Ricci's Lezioni sulla Teoria delle Superficie, and is there applied to the ordinary surface theory. A sufficient introduction for the purposes of this paper will be found in the paper of Wilson and Moore above mentioned. It is their method that is followed in the deduction of the integrability conditions. It was apparently first given by Ricci in the Lezioni, pp. 87-91.

We shall denote by $V_{m}$ a variety of $m$ dimensions, defined in a euclidean space $P_{n}$ of $n$ dimensions by means of $n-m$ equations

$$
F_{\alpha}\left(x_{1}, x_{2}, \cdots, x_{n}\right)=0 \quad(a=1,2,3, \cdots, n-1) .
$$

* Presented to the Society, December 28, 1921.

+ A. Voss, Zur Theorie der Transformation quadratischer Differentialansdrücke und der Kriimmung höherer Mannigfaltigkeiten, Mathematische Annalen, vol. 16 (1880).

$\ddagger$ Beez, Zeitschrift für Mathematik und Physik, vol. 24 (1879), pp. 1, 65.

$\S$ E. B. Wilson and C. L. E. Moore, Differential geometry of two-dimensional surfaces in hyperspace, Proceedings of the American Academy of Arts and Sciences, vol.52 (1916), p. 269.

|| M. M. G. Ricci and T. Levi-Civita, Méthodes de calcul différential absolu et leurs applications, Mathematische Annalen, vol. 54 (1900-1901), pp. 124-201. This paper will be referred to in what follows by the term Absolute Calculus. 
Let $u_{r}$ be a set of variables such that

$$
x_{i}=x_{i}\left(u_{1}, u_{2}, \cdots, u_{m}\right) \quad(i=1,2,3, \cdots, n)
$$

make the $F$ 's identically zero in the $u$ 's. We have then for the differential of arc in $V_{m}$

in which

$$
d s^{2}=\sum_{r s} a_{r s} d u_{r} d u_{s} \quad(r, 8=1,2,3, \cdots, m)
$$

$$
a_{r s}=a_{s r}=\sum_{i} \frac{\partial x_{i}}{\partial u_{r}} \frac{\partial x_{i}}{\partial u_{s}} \quad(i=1,2,3, \cdots, n) .
$$

In our euclidean $n$ space $P_{n}$ let us denote by $i_{n}$ the unit vector parallel to the $x_{h}$ axis. A variable point in $P_{n}$ is given by the vector $x$ having components $x_{h}$ parallel to the corrdinate axes, or

$$
x=\sum_{h} x_{h} i_{h} .
$$

We shall use subscripts to distinguish between vectors and also to denote covariant differentiation with regard to the fundamental quadratic differential form (1), but when it becomes necessary to use subscripts in both senses on the same letter those used for the latter purpose will always follow those used for the former, and the two kinds of subscripts will be separated by a comma.*

\section{DEDUCTION OF INTEGRABILITY CONDITTONS}

We have

$$
a_{r s}=x_{r} \cdot x_{s} .
$$

Differentiating this covariantly we obtain

$$
x_{r t} \cdot x_{s}+x_{r} \cdot x_{s t}=0,
$$

for the first covariant derivative of the fundamental form is zero. Also the second covariant derivative of a scalar is symmetric so that

$$
x_{r s}=x_{s r},
$$

and from these two sets of equations is easily deduced

$$
x_{r} \cdot x_{s t}=0,
$$

which is to say that $x_{s t}$ is normal to $V_{m}$.

* Although using Clarendon type for vectors seems preferable to the author, no ambiguity arises in the present paper from not doing 10. 
The normal to the variety $F_{\alpha}$ is $\nabla F_{\alpha}$ and we shall denote by $\xi_{\alpha}$ the unit vector in this direction. The $n-m$ vectors $\xi_{\alpha}$ define the euclidean $n-m$ space, $\boldsymbol{P}_{n-m}$, normal to $\nabla_{m}$. The subspace of this defined by the $x_{r s}$ will be called the principal normal space. It is convenient to make use also of another set of vectors $\zeta_{a}$ that may be used to define this $P_{n-n}$. We shall call them the polar set, and they shall be defined by the following equations, from which their geometric relation to the vectors $\xi_{a}$ is at once evident:

$$
\zeta_{a} \cdot \xi_{\beta}=0, \quad \alpha \neq \beta, \quad \zeta_{\alpha} \cdot \xi_{a}=1
$$

We have also the relations

$$
\xi_{\alpha} \cdot x_{r}=0, \quad \zeta_{\alpha} \cdot x_{r}=0 .
$$

Whence by covariant differentiation is obtained

$$
\begin{aligned}
& \xi_{a, r} \cdot x_{s}+\xi_{\alpha} \cdot x_{r s}=0, \\
& \zeta_{\alpha, r} \cdot x_{s}+\zeta_{\alpha} \cdot x_{r s}=0, \\
& \xi_{\alpha, r} \cdot \zeta_{\beta}+\xi_{\alpha} \cdot \zeta_{\beta, r}=0 .
\end{aligned}
$$

Since the $x_{r s}$ are normal to $\nabla_{m}$ they can be expressed in terms of the $\xi_{\alpha}$ or the $\zeta_{a}$. It seems more convenient to use the latter. Hence let

$$
x_{r s}=\sum_{\alpha} b_{\alpha r s} \zeta_{\alpha} .
$$

If this be multiplied by $\xi_{a}$ the following is obtained:

$$
\xi_{a} \cdot x_{r s}=b_{a r s}=-\xi_{a, r} \cdot x_{s},
$$

in which the latter equality is obtained by using (3). In (5) set

$$
\xi_{a} \cdot \zeta_{\beta, r}=\mu_{a \beta r}=-\xi_{a, r} \cdot \zeta_{\beta} .
$$

These two sets of equations may be solved for $\xi_{a, r}$ getting

$$
\xi_{\alpha, r}=-\sum_{t} b_{a r t} x^{t}-\sum_{\beta} \mu_{\alpha \beta r} \xi_{\beta} .
$$


Now differentiate (6) covariantly,

$$
x_{r s i}=\sum_{a} b_{a r s, t} \zeta_{a}+\sum_{a} b_{a r s} \zeta_{a, t} .
$$

The vectors $\zeta$ can of course be expressed in terms of the $\xi$, and hence let

$$
\zeta_{\alpha}=\sum_{\gamma} v_{a r} \xi_{\gamma}
$$

in which $v_{\alpha \gamma}$ is symmetric. Now differentiate (8) covariantly, getting

$$
\zeta_{a, t}=\sum_{\gamma} v_{a \gamma, t} \xi_{\gamma}+\sum_{\gamma} v_{a \gamma} \xi_{\gamma, t}
$$

and finally place these values in the above expression for $x_{r s t}$ and simplify by means of (7). Thus is obtained the equation

$$
x_{r s t}=\sum_{\gamma}\left(\sum_{a} b_{\alpha r s, t} \nu_{\alpha \gamma}+\sum_{a} b_{\alpha r s} \nu_{a \gamma, t}-\sum_{a \beta} b_{\alpha r s} \nu_{\alpha \beta} \mu_{\beta \gamma t}\right) \xi_{\gamma}-\sum_{a \gamma r t} b_{a r s} b_{\gamma t t} \nu_{a \gamma} x^{u t} .
$$

A theorem of the Absolute Calculus gives us the following relation between the third covariant derivatives of a function

$$
x_{r s t}-x_{r t s}=\sum_{u} x^{u}(u r, s t)
$$

in which $(u r, s t)$ is the Riemann symbol of the first kind. Hence when this difference is formed the resulting coefficients of $\xi_{\gamma}$ must be zero and the coefficient of $x^{u}$ must be $(u r, s t)$. Thus are obtained the integrability conditions

$$
\begin{aligned}
& (u r, s t)=\sum_{a \beta} v_{a \beta}\left(b_{a r t} b_{\beta s u}-b_{a r s} b_{\beta t u}\right), \\
& \sum_{\alpha} v_{\alpha \gamma}\left[b_{\alpha r s, t}-b_{\alpha r t, s}\right]=\sum_{\alpha \beta} v_{\alpha \beta}\left(b_{\alpha r s} \mu_{\beta \gamma t}-b_{\alpha r t} \mu_{\beta \gamma s}\right) \\
& -\sum_{a}\left(v_{a \gamma, t} b_{\alpha r s}-v_{\alpha \gamma, s} b_{\alpha r t}\right) .
\end{aligned}
$$

If the defining equations $F_{\alpha}=0$ are so chosen that the $\nabla F_{a}$ are mutually perpendicular vectors, as is done in substance by Ricci, these equations have the simpler form

$$
\begin{gathered}
(u r, s t)=\sum_{\alpha}\left(b_{a r t} b_{a s u}-b_{a r s} b_{\alpha t u}\right), \\
b_{a r s, t}-b_{\alpha r t, s}=\sum_{a}\left(b_{\beta r s} \mu_{\beta a t}-b_{\beta r t} \mu_{\beta a s}\right) .
\end{gathered}
$$




\section{ORTHOGONAL NETS OF CURVES IN THE VARIETY}

In order to discuss curvature we shall introduce an orthogonal $m$-ple, after the manner of the absolute calculus.

Let $\lambda^{r}$ be $m$ functions of the $u$ 's which do not vanish in the neighborhood in which we are interested. By means of the equations

$$
\frac{d u_{1}}{\lambda^{1}}=\frac{d u_{2}}{\lambda^{2}}=\cdots=\frac{d u_{m}}{\lambda^{m}}
$$

they define a family of curves on the variety, one through each point. If all the $\lambda$ 's are multiplied by the same function of the $u$ 's it does not change the family of curves represented, and so in particular we can so choose our $\lambda$ 's that they satisfy the relation

$$
\sum_{r s} a_{r s} \lambda^{r} \lambda^{s}=\sum_{r} \lambda_{r} \lambda^{r}=\sum_{r s} a^{r s} \lambda_{r} \lambda_{s}=1
$$

When this is done the $\lambda^{r}$ are called the contravariant coordinates of the family of curves. In this manner $m$ such families may be defined, orthogonal each to each. Such a set of families of curves is called an orthogonal $m$-ple. Indicating the family by the first subscript we have the following relations between the covariant and contravariant coordinates of the families of an orthogonal $m$-ple:

$$
\sum_{r s} a_{r s} \lambda_{h}^{r} \lambda_{k}^{s}=\sum_{r} \lambda_{h r} \lambda_{k}^{r}=\sum_{r s} a^{r s} \lambda_{h r} \lambda_{k s}= \begin{cases}0 & k \neq h, \\ 1 & k=h .\end{cases}
$$

The orthogonal $m$-ple has the following invariants,

$$
I_{\text {ahk }}=\sum_{r s} b_{\text {ars }} \lambda_{h}^{r} \lambda_{k}^{s},
$$

by means of which the $b_{\text {ars }}$ may be written in terms of the $m$-ple

$$
b_{a r s}=\sum_{h k} I_{a h k} \lambda_{k r} \lambda_{k s}
$$

Denote by $b_{\alpha}$ the quadratic differential form

$$
b_{\alpha}=\sum_{r s} b_{\text {ars }} d u_{r} d u_{s}
$$

These forms correspond to the second fundamental form in surface theory. They may be combined into a vector form 


$$
b=\sum_{a} b_{a} \zeta_{a}=\sum_{r s} x_{r s} d u_{r} d u_{s},
$$

which will be known as the second fundamental form of the variety $V_{m}$ in what follows. In terms of the $m$-ple

$$
b=\sum_{n k r s} I_{h k} \lambda_{h r} \lambda_{k s} d u_{r} d u_{s},
$$

in which

we also find

$$
I_{l k k}=\sum_{a} I_{a h k} \zeta_{a},
$$

$$
x_{r s}=\sum_{h r} I_{h k} \lambda_{l i r} \lambda_{k s} .
$$

\section{CURVature OF THE VARIETY}

The unit vectors tangent to the curves $\lambda_{h}^{r}$ are

$$
\eta_{h}=\sum_{r} \lambda_{h}^{r} x_{r}
$$

and since the curves are those of an orthogonal $m$-ple these vectors are mutually perpendicular. The rate of change of these vectors may be found by covariant differentiation

$$
\eta_{h, r}=\sum_{s} \lambda_{h}^{s} x_{r s}+\sum_{s} \lambda_{h s, r} x^{s} .
$$

Now by Absolute Calculus, p. 149,

$$
\lambda_{h r, s}=\sum_{i s} \gamma_{h i j} \lambda_{i r} \lambda_{j s},
$$

and if we substitute this and (15) in the above we get

$$
\eta_{h, r}=\sum_{i} I_{i h} \lambda_{i r}+\sum_{i j} \gamma_{h i j} \lambda_{j r} \eta_{i} .
$$

Now the curvature of the curves $\lambda_{h}^{r}$ is

$$
\begin{aligned}
K_{h}=\frac{d \eta_{h}}{d s_{h}}=\sum_{r} \eta_{h, r} \frac{d u_{r}}{d s_{h}}=\sum_{r} \eta_{h, r} \hat{\lambda}_{h h}^{r} & =I_{h h}+\sum_{i} \gamma_{h i h} \eta_{i} \\
& =I_{h h}-\sum_{i} \gamma_{i h h} \eta_{i} .
\end{aligned}
$$


Also* $\gamma_{i h h}=0$ is the condition that the family of curves $\lambda_{h}^{r}$ be geodesics. Hence the curvature of a curve on the variety consists of two parts, a vector part $I_{h h}$ normal to the variety, which is the same for all curves having the same direction and so may be considered as the normal curvature of the variety in that direction, and a vector part in the variety which measures the deviation from the geodesic in that direction, and so is the geodesic curvature of the curve.

By an argument similar to that used by Wilson and Mooref one easily proves the two theorems:

If a curve is projected on any $P_{k}$ through the tangent line to the curve, the curvature of the projection at the point of tangency is equal to the projection of the curvature at that point.

The projection of the curvature of any section of the variety made by a $P_{n-m+1}$ on the normal $P_{n-n+1}$ passing through the same direction on the variety is equal to the curvature of the normal section.

If we compute the rate of change of any unit normal, in the variety, to our curve, we have

$$
\frac{d \eta_{k}}{d s_{h}}=\sum_{r} \eta_{k, r} \lambda_{h}^{r}=I_{h k}-\sum_{i} \gamma_{i h k} \eta_{i}
$$

which gives the interpretation of the invariants $I_{h k}$.

If the invariant of the variety $\sum_{r s} a^{r s} x_{r s}$ be computed in terms of the invariants of the net it is found that

$$
\sum_{r s} a^{r s} x_{r s}=\sum_{h k} a^{r s} I_{h k} \lambda_{h r} \lambda_{k s}=\sum_{h} I_{h h}
$$

In other words, the sum of the normal curvatures in any $m$ mutually orthogonal directions in the variety is independent of the directions. It is then a numerical measure of the curvature of the variety, being a natural extension of the notion of mean curvature of a surface in three dimensions. We shall set

$$
h=\frac{1}{m} \sum_{h} I_{h h}=\frac{1}{m} \sum_{r s} a^{r s} x_{r s},
$$

and shall call $h$ the mean vector curvature of the variety.

* Absolute Calculus, p. 154.

† Loc. cit., p. 320 and p. 321. 


\section{CuRvature OF the variety WITH RESPECT TO A GIVEN NORMAL DIRECTION}

The above vector $h$ may be obtained in several different ways, some of which seem to help in forming a conception of the geometry of the situation. We shall take up first the notion of curvature with respect to a given normal direction. In ordinary surface theory the normal curvature of a surface may be found for any direction through the surface point by taking a neighboring point in that direction and finding its distance from the tangent plane. Calling this distance $p$, the curvature is

$$
K=\frac{2 p}{d s^{2}},
$$

which may be put into vector notation

$$
K=-\frac{d n \cdot d x}{d s^{2}}=-\frac{d n \cdot d x}{d x \cdot d x}
$$

Similarly, at any point of our variety we may choose a normal direction and define it by a unit vector $\sigma$. Through the variety point there is a unique $\boldsymbol{P}_{n-1}$ perpendicular to $\sigma$ and it is of course tangent to the variety. If we take for our $p$ in the above formula the perpendicular distance from a neighboring point in the variety to this $P_{n-1}$, we have defined curvature with respect to the direction $\sigma$. Written in terms of vectors this definition gives

$$
K_{\sigma}=-\frac{d \sigma \cdot d x}{d x \cdot d x}=-\sum_{r s} \sigma_{r} \cdot x_{s} \lambda r \lambda^{s} .
$$

We now determine those directions in the variety for which $K$ is stationary, which we shall call the directions of principal curvature of the variety with respect to the direction $\sigma$. Since $\sigma$ is perpendicular to the variety, $\sigma \cdot x_{r}=0$, and by covariant differentiation

$$
\sigma_{s} \cdot x_{r}+\sigma \cdot x_{r s}=0,
$$

so that the curvature may be written in the form

$$
K=-\sum_{r s} \sigma_{r} \cdot x_{s} \lambda^{r} \lambda^{s}=\sum_{r s} \sigma \cdot x_{r s} \lambda^{r} \lambda^{s}=\frac{\sigma \cdot b}{d x \cdot d x} .
$$


By differentiation of the second expression for $K$, remembering that the $\lambda$ 's are connected by the relation (11), we find that the required directions are given by

$$
\sum_{s}\left(\sigma \cdot x_{r s}-\varrho a_{r s}\right) \lambda^{s}=0
$$

in which $\varrho$ is a root of the determinantal equation

$$
\left|\sigma \cdot x_{r s}-\rho a_{r s}\right|=0 \text {. }
$$

From the form of these equations we know that these directions are mutually orthogonal, and so form an orthogonal $m$-ple which we shall call the $m$-ple of curvature with respect to the direction $\sigma$. Using this $m$-ple as $m$-ple of reference we have*

$$
\sigma \cdot x_{r s}=\sum_{h} \varrho_{h} \lambda_{h r} \cdot \lambda_{h s}
$$

where the $\rho$ 's are the roots of (21). Now (15) gives $x_{r \cdot s}$ in terms of any $m$-ple, so for this particular one

$$
\sigma \cdot I_{h k}:=0, \quad k \neq h, \quad \varrho_{h}=\sigma \cdot I_{h h} .
$$

Moreover the principal curvatures are precisely the $\varrho_{h}$, so that we have the theorem:

The curvature of a curve of the m-ple of curvature with respect to the direction $\sigma$ is the projection of its vector normal curvature on the direction $\sigma$.

The mean of these principal curvatures is given by

$$
\frac{1}{n \imath} \sum_{h} \varrho_{h}=\frac{1}{m} \sum_{r s} a^{r s} \sigma \cdot x_{r s}=\sigma \cdot h .
$$

For a particular direction $\sigma$, this mean curvature has a maximum, which may be found by differentiation and when so found turns out to be precisely the direction $h$. The $m$-ple of curvature with regard to this direction we call the principal $m$-ple of curvature of the variety.

From the above expression for the mean curvature it is obvious that the mean curvature of the variety with respect to any direction $\sigma$ perpendicular to $h$ is zero.

Many of the theorems of ordinary differential geometry may be extended by means of this notion of curvature with respect to a given normal direction $\sigma$, for example:

* Absolute Calculus, p. 159. 
If the normals, $\sigma$, along any curve form a developable then the curve is a line of curvature with respect to the direction $\sigma$.

The proof is as follows

$$
Y=x+t \sigma
$$

is any point on such a normal. If we take a similar line at an infinitesimal distance along any curve $\lambda^{r}$ we have

$$
d Y=d x+t d \sigma+\sigma d t .
$$

If these lines are to form a developable there must be some value of $t$ for which $d Y$ has the same direction as $\sigma$. For this value

$$
d x+t d \sigma+\sigma d t=k \sigma .
$$

And if we multiply by $\sigma$ we find

whence

$$
d t=k,
$$

which gives

$$
d x+t d \sigma=0,
$$

$$
\sum_{r}\left(x_{r}+t \sigma_{r}\right) \lambda^{t}=0
$$

and multiplying by $x_{s}$ we obtain

$$
\sum_{r}\left(a_{r s}+t \sigma_{r} \cdot x_{s}\right) \lambda_{r}^{r}=0 .
$$

But since $\sigma \cdot x_{r}=0$ it follows that

$$
\sigma_{r} \cdot x_{s}+\sigma \cdot x_{r s}=0
$$

and this further simplifies our equation to

$$
\sum_{r}\left(a_{r s}-t \sigma \cdot x_{r s}\right) 2^{r}=0,
$$

which is equivalent to (20) and so proves the theorem.

\section{The ASYMPTO'TIC CONE AND THE DUPIN INDICATRIX}

We may call the directions in which the tangent $P_{n-1}$ perpendicular to $\sigma$ intersects $V_{m}$ the asymptotic directions with respect to the direction $\sigma$. These directions form a second degree cone of $m-1$ dimensions which we call the 
asymptotic cone. Similarly, if this $\boldsymbol{P}_{\boldsymbol{n}-1}$ is infinitesimally displaced but kept normal to $\sigma$ it will intersect $V_{m}$ in a quadratic manifold which we shall call the Dupin indicatrix with respect to the direction $\sigma$. When the direction $\sigma$ is that of $h$ we call the asymptotic cone the principal asymptotic cone and the indicatrix the principal Dupin indicatrix.

Analytioally

$$
(X-x) \cdot \sigma=0
$$

is the tangent $P_{n-1}$. Displaced slightly it becomes

$$
(X-x) \cdot \sigma=\varepsilon
$$

If $V_{m}$ is expanded in the neighborhood of the point $x$ we have

$$
X=x+\sum_{r} \frac{\partial x}{\partial u_{r}}\left(u_{r}-u_{r}^{0}\right)+\frac{1}{2} \sum_{r s} \frac{\partial^{2} x}{\partial u_{r} \partial u_{s}}\left(u_{r}-u_{r}^{0}\right)\left(u_{s}-u_{s}^{0}\right)+\cdots
$$

and we have for the intersection

$$
\frac{1}{2}\left[\sum_{r s} \frac{\partial^{2} x}{\partial u_{r} \partial u_{s}}\left(u_{r}-u_{r}^{0}\right)\left(u_{s}-u_{s}^{0}\right)\right] \cdot \sigma=\varepsilon,
$$

which simplifies to

in which

$$
\sum_{r s} \sigma \cdot x_{r s} v_{r} v_{s}=\varepsilon
$$

$$
v_{r}=u_{r}-u_{r}^{0}
$$

If we wish to replace the $v_{r}$ by variables measured along an arbitrary orthogonal $m$-ple we can write the indicatrix as

$$
\sum_{i j} \sigma \cdot I_{i j} w_{i} w_{j}=8
$$

The asymptotic cone with regard to the direction $\sigma$ is thus defined by

$$
\sigma \cdot b=0
$$

and the principal asymptotic cone by

$$
h \cdot b=0
$$


We shall call a curve on $V_{m}$ an asymptotic with respect to the direction $\sigma$ if its tangent at every point belongs to the asymptotic cone with respect to the direction $\sigma$ at that point.

If we introduce an orthogonal $m$-ple we find that the condition that the curve $\lambda_{m}^{r}$ be an asymptotic is

$$
\sigma \cdot I_{m m}=0
$$

whence we conclude:

$A$ necessary and sufficient condition that a curve on $V_{m}$ be an asymptotic with respect to the direction $\sigma$ is that its curvature be normal to $\sigma$.

From which follows immediately:

An asymptotic with respect to the direction $\sigma$ is characterized by the property that its osculating plane is normal to $\sigma$.

The discriminant of the Dupin indicatrix is

$$
\left|\sigma \cdot x_{r s}\right|=0
$$

Whence the lines of this cone are the directions in space with respect to which the asymptotic cone has multiple elements.

Another theorem seems worthy of note:

If an orthogonal m-ple can be picked from the principal asymptotic directions, then $V_{m}$ is a minimal variety.

For taking this $m$-ple as $m$-ple of reference

or

$$
\sum_{r s} h \cdot x_{r s} \lambda_{i}^{r} \lambda_{i}^{s}=0
$$

$$
h \cdot I_{i i}=0
$$

for every $i$. But $h$ is a linear combination of the $I_{i i}$ and so this relation cannot hold unless $h=0$. On the other hand $h=0$ is precisely the condition for a minimal variety.*

\section{CONJUGate directions}

Let us denote by $\varphi$ the dyadic

$$
\varphi \equiv \sum_{i j} \sigma \cdot I_{i j} \eta_{i} \eta_{j}
$$

* C. L. E. Moore, Note on minimal varieties in hyperspace, Bulletin of the American Yathematical Society, rol. 27 (1921), p. 216. 
Then if $r=\sum_{i} w_{i} \eta_{i}$, the indicatrix may be written

$$
\boldsymbol{r} \cdot \boldsymbol{\varphi} \cdot \boldsymbol{r}=\boldsymbol{\varepsilon}
$$

Now let $\eta$ be any given vector in the tangent $\boldsymbol{P}_{\boldsymbol{m}}$, and $\boldsymbol{\tau}$ a variable vector also in it. The line $\tau+s \eta$ will intersect the indicatrix in points determined by

$$
(\tau+s \eta) \cdot \varphi \cdot(\tau+s \eta)=\varepsilon .
$$

If now $\tau$ is the vector to the mid point of the chord, then the coefficient of the first degree term in $s$ must be zero, or

$$
\boldsymbol{x} \cdot \boldsymbol{\varphi} \cdot \eta=0
$$

defines the locus of the mid points of the chords of $\varphi$ that are parallel to $\eta$. It defines in the tangent $\boldsymbol{P}_{\boldsymbol{m}}$ a $\boldsymbol{P}_{\boldsymbol{m}-1}$.

A set of $m$ directions such that any $m-1$ of them determine the $P_{m-1}$ so related to the remaining one we shall call a conjugate set of directions with respect to the direction $\sigma$. If we have an $m$-ple of curves on $V_{m}$ such that their directions at every point form a conjugate set of directions with respect to the direction $\sigma$, then we say that we have a conjugate $m$-ple with respect to the direction $\sigma$.

The above $P_{m-1}$ may be obtained in another way. If we take consecutive tangent $\boldsymbol{P}_{n-1}$ 's normal to $\sigma$ and in the direction $\eta$, a point of intersection is defined by

$$
\tau \cdot d \sigma=0
$$

which can easily be reduced to the form

or

$$
\boldsymbol{\tau} \cdot \boldsymbol{\varphi} \cdot \eta=0
$$

This $P_{m-1}$ is the locus of points of intersection of consecutive tangent $P_{n-1}$ 's normal to $\sigma$ in the direction $\eta$.

Finally, the lines of curvature with respect to the direction $\sigma$ are the only conjugate orthogonal $m$-ple with respect to the direction.

For let us assume an orthogonal conjugate $m$-ple and let $\eta_{i}$ be unit vectors in these directions. Then

$$
\varphi=\sum_{i j} \sigma \cdot I_{i j} \eta_{i} \eta_{j}
$$

Since $\eta_{i}$ and $\eta_{j}$ are conjugate, $\eta_{i} \cdot \varphi \cdot \eta_{j}=0$, whence $\sigma \cdot I_{i j}=0, i \neq j$, and this is given in (22) as the condition that the lines of curvature are the coordinate $m$-ple. 


\section{The osculating $\boldsymbol{P}_{\boldsymbol{m}+1}$}

We are led to the direction $h$ in another way which is of interest. Any point in the tangent $\boldsymbol{P}_{m}$ is given by

$$
y=x+\sum_{r} \mu^{r} x_{r},
$$

in which the $\mu_{r}$ are parameters. Likewise if $\sigma$, as before, is a unit vector perpendicular to the variety, any point in the $P_{m+1}$ determined by it and the tangent $\boldsymbol{P}_{m}$ is given by

$$
y=x+\sum_{r} u^{v} x_{r}+\mu \sigma .
$$

Now take any point $z$ of $P_{n}$ not in this $P_{m+1}$. There is through $z$ a unique $P_{n-m-1}$ of which every direction is perpendicular to every direction of the $P_{m+1}$. It intersects the $P_{m+1}$ in just one point, which is then the projection of the given point on the $P_{m+1}$. We may thus project our variety on the $\boldsymbol{P}_{\boldsymbol{m}+1}$. Then in the $\boldsymbol{P}_{\boldsymbol{m}+1}$ there is a unique direction normal to the projection of our variety, and hence the curvature of the projection may be defined in the usual manner. When this is done it turns out to be precisely the quantity that we have found as the curvature of the variety with respect to the direction $\sigma$.

The work is as follows.

The equations of the $P_{n-m-1}$ are

$$
(z-y) \cdot x_{r}=0, \quad(z-y) \cdot \sigma=0,
$$

and to find the point of intersection with the $P_{m+1}$ we substitute the value of $y$ from (23) in these and solve for $\mu$, getting

$$
\mu=(z-x) \cdot \sigma .
$$

Since $\sigma$ is a unit vector perpendicular to $P_{m}$, the perpendicular distance from the projection of $z$ to $P_{m}$ is $\mu$.

Now let $z$ be a neighboring point of the variety

$$
z=x+d x+\frac{1}{2} d^{2} x .
$$

Then its projection is a neighboring point of the projected variety and the distance of its projection from $P_{m}$ becomes, by the above formula for $\mu$ and the fact that $\sigma \cdot d x=0$, equal to

$$
\mu=\frac{1}{2} \sum_{r s} \sigma \cdot x_{r s} d u_{r} d u_{s} .
$$


Hence the curvature of the projected variety is

$$
K=\frac{2 \mu}{d s^{2}}=\sum_{r s} \sigma \cdot x_{r s} \lambda^{r} \lambda^{s},
$$

which is just the form we deduced from (19).

We now may ask, of all $P_{m+1}$ 's through the tangent $P_{m}$ which fits the variety best? If we define best fit by saying that the mean curvature of the projection of the variety on the $P_{m+1}$ shall be a maximum, we find that the answer is that $\boldsymbol{P}_{\boldsymbol{m}+1}$ which is determined by the vector $h$. This $\boldsymbol{P}_{\boldsymbol{m + 1}}$ we shall call the osculating $P_{m+1}$ to the given variety.

One can go ahead and define similarly an osculating $\boldsymbol{P}_{\boldsymbol{m}+2}$, but that would involve third derivatives and will not be considered in this paper.

We remember that the normal curvature of the variety in the direction $\lambda_{h}^{r}$ is $I_{h h}$, and by solving (15) we get

$$
I_{h h}=\sum_{r s} x_{r s} \lambda_{h}^{r} \lambda_{h}^{s}
$$

Also by (24) the curvature of the projection of a curve having this direction, on the $P_{m+1}$ through the tangent line defined by the direction $\lambda_{h}^{r}$, is

$$
K=\sum_{r s} \sigma \cdot x_{r s} \lambda_{h}^{r} \lambda_{h}^{s}=\sigma \cdot I_{h h},
$$

and since the projection of $I_{h h}$ on the direction $\sigma$ is $\sigma \cdot I_{h h}$ we have the theorem:

The projection of the mirvature at the point of tangency of any morve of the variety on any $P_{m+1}$ through the tangent $P_{m}$ is equal to the curvature of the projection of the curve.

\section{CanONical aXes fOR THE PRINCIPAL NORMaL SPACE}

The principal curvatures with regard to the direction $\sigma$ are the roots of (21). If we write this out as a polynomial in $\varrho$, and make the coefficient of the highest power of $\varrho$ unity, then the other coefficients are the invariants that correspond to mean and total curvature in surface theory. We have already made use of the coefficient of $e^{m-1}$ in our mean curvature. Its maximum gave us the direction $h$. We shall now make use of the coefficient of $\rho^{m-2}$ which is the sum of the products of the principal curvatures with respect to the direction $\sigma$ taken two at a time. The other coefficients, for whose simple treatment some elements of a theory of polyadics is desirable, will not be discussed in this paper. We now ask for what directions $\sigma$ has this coefficient of $\rho^{m-2}$ stationary values? We find that there are $m(m+1) / 2$ such directions, all mutually perpendicular. The work is as follows: 
Denote the coefficient in question by $D_{2}$. It may be written

$$
D_{\mathbf{q}}=\frac{1}{4} \sum_{i j k e}\left|\begin{array}{ll}
\sigma \cdot x_{i j} & \sigma \cdot x_{i e} \\
\sigma \cdot x_{k j} & \sigma \cdot x_{k e}
\end{array}\right| \cdot\left|\begin{array}{ll}
a^{i j} & a^{i e} \\
a^{k j} & a^{k e}
\end{array}\right| .
$$

If this is expanded and the values of $x_{r s}$ in terms of the $I_{h k}$ are substituted,

$$
D_{\mathbf{q}}=\frac{1}{2}\left\{\left[\sum_{h} \sigma \cdot I_{h h}\right]^{2}-\sum_{h k}\left(\sigma \cdot I_{h k}\right)^{2}\right\}
$$

is obtained. This in turn may be written

in which $\psi$ is the dyadic

$$
D_{2}=\sigma \cdot \psi \cdot \sigma,
$$

$$
2 \psi^{\prime}=m^{2} h h-\sum_{h k} I_{h k} I_{h k}=\sum_{h k}\left(I_{h h} I_{k k}-I_{h k} I_{h k}\right) .
$$

To find the stationary values of $D_{2}$ we have

$$
D_{2}=\sigma \cdot \psi \cdot \sigma \text { and } \sigma \cdot \sigma=1
$$

and differentiating these we get

$$
\sigma \cdot \psi \cdot d \sigma=0 \text { and } \sigma \cdot d \sigma=0,
$$

from which $d \sigma$ must be eliminated. The second equation tells us that we must choose $d \sigma$ so that it is perpendicular to $\sigma$ while the first says that for any such choice the vector $\sigma \cdot \psi$ must be perpendicular to $d \sigma$. Hence the vectors $\sigma \cdot \psi$ and $\sigma$ must have the same direction, or

$$
\sigma \cdot \psi=k \sigma .
$$

This defines in the principal normal space the axes of the hyperquadric

$$
\sigma \cdot \psi \cdot \sigma=\text { const. }
$$

From (27), by multiplying by $\sigma$, we find

$$
\sigma \cdot \psi \cdot \sigma=k,
$$

so that the values of $k$ are the stationary values of $D_{\mathbf{2}}$.

Now set

$$
\sigma=\sum_{r s} \sigma_{r s} I_{r s}^{\prime},
$$

in which $I_{r s}^{\prime}$ are the polar set of vectors $I_{r s}, i$. e., $I_{i j}^{\prime}$ is in the space determined by the $I$ 's and is perpendicular to all the $I$ 's except $I_{i j}$, and 


$$
\begin{array}{ll}
I_{r s}^{\prime} \cdot I_{t u}=0 & (r, s \neq t, u), \\
I_{r s}^{\prime} \cdot I_{r s}=\frac{1}{2} & (r \neq s), \\
I_{r r}^{\prime} \cdot I_{r r}=1 . &
\end{array}
$$

Multiplying this relation by $I_{r s}$ we get

$$
\sigma_{r s}=\sigma \cdot I_{r s},
$$

and substituting these in (27) gives

$$
\sum_{r s} \sigma_{r s} I_{r s}^{\prime} \cdot \psi \cdot I_{t u}-k \sigma_{t u}=0,
$$

so that the values of $k$ are given by the equation

$$
\begin{aligned}
\left|I_{r s}^{\prime} \cdot \psi \cdot I_{t u}-k \varepsilon_{r s, t u}\right|=0, & r \leqq s, t \leqq u, \\
\varepsilon_{r s, t u}=0 & (r, s \neq t, u), \\
\varepsilon_{r s, r s}=\frac{1}{2} & (r \neq s), \\
\varepsilon_{r r, r r}=1 . &
\end{aligned}
$$

We can find the sum of the $k$ 's by expanding this and taking the coefficient of the second highest power of $k$, and this gives

$$
\sum_{r \leq s} k_{r s}=\sum_{r \leq s} I_{r s}^{\prime} \cdot \psi \cdot I_{r s}
$$

from which we easily deduce

$$
\sum_{r \leq s} k_{r s}=\frac{1}{2} G=\psi_{s}=\frac{1}{2} \sum_{r s t u} a^{u s} a^{r t}(u r, s t) .
$$

This is called the Gaussian curvature of the variety. It is to be noted that it is the scalar of the dyadic $\psi$.

The directions $\sigma$ defined by equation (28) form a mutually orthogonal system, which may be used as a canonical system of axes for the principal normal space.

$$
\text { 10. The tangent } P_{m}
$$

We may write the unit tangent $\boldsymbol{P}_{m}$ as

whence

$$
P_{m}=\eta_{1} \times \eta_{2} \times \eta_{3} \times \cdots \times \eta_{m},
$$

but

$$
d P_{m}=\sum d \eta_{1} \times \eta_{2} \times \cdots \times \eta_{m},
$$

$$
d \eta_{i}=\sum_{r} \eta_{i, r} d u_{r}=\sum_{r, j} I_{j i} \lambda_{j r} d u_{r}+\sum_{r j k} \gamma_{i j k} \lambda_{k r} \eta_{j} d u_{r}
$$

in which we must remember that* $\gamma_{h h e}=0$.

* Absolute Calculus, p. 149. 
And so we have

$$
d P_{m}=\sum_{r j}\left(\sum I_{j 1} \times \eta_{2} \times \cdots \times \eta_{m}\right) \lambda_{j r} d u_{r}
$$

since the latter part drops, due to having two identical $\eta$ 's in each term. Now

$$
d x=\sum_{r} x_{r} d u_{r}
$$

and

$$
\eta_{h}=\sum_{r} \lambda_{h}^{r} x_{r}
$$

and solving this

$$
x_{s}=\sum_{h} \lambda_{h s} \eta_{h}
$$

whence

$$
d x=\sum_{s, h} \lambda_{h s} \eta_{h} d u_{s} .
$$

So finally we get the formula

$$
\begin{aligned}
d x \times d P_{m} & =\sum_{s h r j}\left(\sum \eta_{h} \times I_{j 1} \times \eta_{2} \times \cdots \times \eta_{m}\right) \lambda_{j r} \lambda_{h s} d u_{r} d u_{s} \\
& =(-1)^{m-1} P_{m} \times \sum_{r s} x_{r s} d u_{r} d u_{s}, \\
& =(-1)^{m-1} P_{m} \times b .
\end{aligned}
$$

We are using the rules of $n$-dimensional vector algebra as given by Lewis.* From these rules one easily deduces the following relation between dot and cross products of two mth order vectors and one first order vector. The subscripts indicate order:

$$
t_{m} \cdot\left(r_{1} \times s_{m}\right)=\left(t_{m} \cdot s_{m}\right) r_{1}+\left(r_{1} \cdot t_{m}\right) \cdot s_{m}
$$

We dot-multiply $\boldsymbol{P}_{m}$ into our previous formula and apply this relation:

$$
\begin{gathered}
\boldsymbol{P}_{m} \cdot\left(d x \times d \boldsymbol{P}_{m}\right)=\boldsymbol{P}_{m} \cdot\left(\boldsymbol{P}_{m} \times b\right)(-1)^{m-1}=-\boldsymbol{P}_{m} \cdot\left(b \times \boldsymbol{P}_{m}\right) \\
\left(\boldsymbol{P}_{m} \cdot d \boldsymbol{P}_{m}\right) d x+\left(d x \cdot \boldsymbol{P}_{m}\right) \cdot d \boldsymbol{P}_{m}=-\left(\boldsymbol{P}_{m} \cdot \boldsymbol{P}_{m}\right) b-\left(b \cdot \boldsymbol{P}_{m}\right) \cdot \boldsymbol{P}_{m} .
\end{gathered}
$$

Now

$$
P_{m} \cdot d P_{m}=0, \quad P_{m} \cdot P_{m}=1, \quad P_{m} \cdot b=0
$$

* Gilbert N. Lewis, On four-dimensional vector analysis, and its application in electrical theory, Proceedings of the American Academ of Arts and Sciences, vol. 46 (1910), pp. $168-169$. 
Hence the above simplifies to

$$
\left(d x \cdot P_{m}\right) \cdot d P_{m}=-b
$$

which is the natural extension of the similar formula of surface theory. The quantity $d x \cdot P_{m}$ is a vector of order $m-1$ in the tangent $\boldsymbol{P}_{m}$ and perpendicular to $d x$.

We have thus the expression of the vector second fundamental form in terms of the tangent plane and its differential.

Another formula that we want is that for $d P_{m} \cdot d P_{m}$. We have

$$
d P_{m} \cdot d P_{m}=\left[\sum_{r j}\left(\sum I_{j 1} \times \eta_{2} \times \cdots \times \eta_{m}\right) \lambda_{j r} d u_{r}\right]^{2}
$$

When this is multiplied out we have terms of the type

$$
\begin{aligned}
\left(\eta_{1} \times \eta_{2} \times \cdots \times \eta_{i-1} \times I_{k i} \times \eta_{i+1}\right. & \left.\times \cdots \times \eta_{m}\right) \\
& \cdot\left(\eta_{1} \times \eta_{2} \times \cdots \times \eta_{j-1} \times I_{h j} \times \eta_{j+1} \times \cdots \times \eta_{m}\right),
\end{aligned}
$$

which are zero unless the $\eta$ missing from each factor is the same, in which case we have $I_{k i} \cdot I_{h i}$.

So

$$
d P_{m} \cdot d P_{m}=\sum_{i h k r s} I_{h i} \cdot I_{k i} \lambda_{h r} \lambda_{k s} d u_{r} d u_{s}
$$

or

$$
d P_{m} \cdot d P_{m}=\sum_{r s} U_{r s} d u_{r} d u_{s}
$$

in which

$$
U_{r s}=\sum_{i h k} I_{h i} \cdot I_{k i} \lambda_{k r} \lambda_{k s}=\sum_{p q} a^{p q} x_{p r} \cdot x_{q s}
$$

are invariants of the variety, which here have their geometric interpretation.

There is another set of invariants of the variety which we wish to use. They are

If now we form

$$
T_{r s}=\sum_{p q} a^{p q} x_{p q} \cdot x_{r s}=m h \cdot x_{p q}
$$

$$
\sum_{r s} T_{r s} d u_{r} d u_{s}=m h \cdot b,
$$

we see from (19) that this is the curvature of the variety with respect to the direction $h$, or rather, a multiple of it. The relation $T_{r s}=0, r \neq s$ is the condition that the principal $m$-ple of curvature be taken for the parametric curves, but, of course, it cannot always be done. 


\section{The Einstein equations}

If now we set

we find that

$$
G_{r s}=U_{r s}-T_{r s}
$$

$$
G_{r s}=\sum_{p q} a^{p q}(r p, q s)
$$

from the integrability conditions. But $G_{r s}=0$ are precisely the Einstein equations for material free space. From the work above we have

$$
\sum_{r s} G_{r s} d u_{r} d u_{s}=d P_{n} \cdot d P_{m}-m h \cdot b,
$$

and the Einstein conditions mean that this expression on the left is zero for all directions in the variety. A variety for which this is true we shall call an Einstein variety. For such a variety, $\frac{d P_{m} \cdot d P_{m}}{d s^{2}}=\frac{m h \cdot b}{d s^{2}}$.

So we have the theorem:

An Einstein variety is a variety for which the square of the vector rate of change of the tanyent $P_{m}$ for any direction in the variety is equal to $1 /(m h)$ times the normal curvature of the variety in that direction. In this statement $h$ is the scalar of the vector $h$.

For the principal asymptotic directions (34) becomes

$$
d P_{n} \cdot d P_{m}=\sum_{r s} G_{r s} d u_{r} d u_{s}
$$

or the rate of turning of the tangent $P_{m}$ along a principal asymptotic direction is

$$
\left(\frac{d P_{m}}{d s}\right)^{2}=\frac{\sum_{r s} G_{r s} d u_{r} d u_{s}}{\sum_{r s} a_{r s} d u_{r} d u_{s}} .
$$

\section{ThE INDICATRIX}

The normal curvature of the variety in any direction is a vector in the normal $P_{n-m}$ at the point in question. If we take the locus of the end of this vector for all directions in the variety emanating from the point, we get in the normal $P_{n-m}$ a variety which we shall call the indicatrix of our variety $V_{m}$ for the point.

To study this locus we shall introduce a variable orthogonal $m$-ple. Let

$$
\mu_{k r}=\sum_{h} c_{h k} \lambda_{h r}
$$


whence

$$
\lambda_{h}^{s}=\sum_{k} c_{h k} \mu_{k}^{s}, \quad \lambda_{h s}=\sum_{k} c_{h k} \mu_{h s}
$$

and since this is an orthogonal transformation

$$
\sum_{h} c_{h k} c_{h e}=\left\{\begin{array}{ll}
0 & k \neq e \\
1 & k=e
\end{array}\right\}=\sum_{h} c_{k h l} c_{e h} .
$$

Solving (15) we obtain

whence

$$
I_{h k}=\sum_{r s} \lambda_{h}^{r} \lambda_{k}^{s} x_{r s}
$$

$$
\begin{aligned}
I_{h k} & =\sum_{r s m n} c_{h m} c_{k n} \mu_{m}^{n} \mu_{n}^{s} x_{r s} \\
& =\sum_{m n} c_{h m} c_{k n} J_{m n},
\end{aligned}
$$

in which we have denoted by $J_{m n}$ the corresponding invariants of the $m$-ple $\mu$. Solving these last equations we get

$$
J_{i j}=\sum_{h k} c_{h i} c_{k j} I_{h k} \text {. }
$$

The vectors $J_{i j}$ therefore lie in the plane space determined by the $I_{i j}$. If the $I_{i j}$ are all independent vectors this space is of dimensionality $m+\frac{1}{2} m(m-1)$ $=\frac{1}{2} m(m+1)$. We shall assume that this is the case, so that $\frac{1}{2} m(m-1)$ is less than $n-m$ in our discussion.

In this case there is a system of vectors $I_{h k}^{\prime}$ polar to the $I_{h k}$. There is also a set of vectors $J_{h k}^{\prime}$ polar to the $J_{h k}$. A little computation shows that the transformation from the $I_{h k}^{\prime}$ to the $J_{h k}^{\prime}$ is precisely (35).

From the relation (35) we see that the indicatrix is defined parametrically by the equation

$$
J=\sum_{h k} c_{h} c_{k} I_{h k}, \text { in which } \sum_{h} c_{h}^{2}=1
$$

If we wish to define it implicitly we shall find it convenient to make use of the system of vectors $x_{r s}^{\prime}$ polar to the system $x_{r s}$. We have

whence

$$
x_{r s}^{\prime}=\sum_{p q}\left(x_{r s}^{\prime} \cdot I_{p q}\right) I_{p q}^{\prime}
$$

$$
x_{r s}^{\prime}=\sum_{p q} \lambda_{p}^{r} \lambda_{q}^{s} \cdot I_{p q}^{\prime}
$$

so that

$$
I_{p q}^{\prime}=\sum_{r s} x_{r s}^{\prime} \lambda_{p r} \lambda_{q s}
$$


If we denote by $\psi_{i j}^{\prime}$ the determinant

$$
\psi_{i j}^{\prime} \equiv\left|\begin{array}{cc}
x_{i i}^{\prime} & x_{i j}^{\prime} \\
x_{j i}^{\prime} & x_{j j}^{\prime}
\end{array}\right|,
$$

then by direct substitution

$$
I_{h h} \cdot \psi_{i j}^{\prime} \cdot I_{h h}=0 \quad(i, j=1,2, \cdots, m)
$$

so that the indicatrix lies on all the quadratic hypercones

$$
\sigma \cdot \psi_{i j}^{\prime} \cdot \sigma=0
$$

These, with the hyper lane

$$
m h^{\prime} \cdot \sigma=1,
$$

completely define it, and are equivalent to (36). In this, $h^{\prime}$ is the vector formed by substituting $I_{i j}^{\prime}$ for $I_{i j}$ in the formula for $h$. The count of the dimensionality of the indicatrix from either of these representations gives $m-1$, as is also obvious from the definition.

If we denote by $\psi^{\prime}$ the dyadic formed by substituting $I_{i j}^{\prime}$ for $I_{i j}$ in the formula for $\psi$, we have

$$
h^{\prime}=\frac{2}{m-1} h \cdot \psi^{\prime}
$$

The determinantal equation (21) may be multiplied by the non-vanishing determinants $\left|\lambda_{q}^{r}\right| \cdot\left|\lambda_{p}^{s}\right|=\left|a_{r s}\right|$ to give the form

$$
\left|\sigma \cdot I_{h k}-\varrho \varepsilon_{h k}\right|=0, \quad \varepsilon_{h k}=\left\{\begin{array}{ll}
0 & h \neq k \\
1 & h=k
\end{array} .\right.
$$

From this form we get the $D_{i}$ immediately in terms of the $I_{i j}$. These, as well as the corresponding equations in the $I_{i j}^{\prime}$, which we shall denote by $D_{i}^{\prime}$, are invariants of the orthogonal substitution (35). Now in the space determined by the $I_{i j}$

$$
D_{1}^{\prime}(\sigma) \equiv m \sigma \cdot h^{\prime}=1
$$

is a hyperplane through the end of $h$ and contains the indicatrix. It is in fact the equation (41). Also

$$
D_{i}^{\prime}(\sigma)=0 \quad(i=2,3, \cdots, m)
$$

are cones of degrees from 2 to $m$ with vertices at the variety point and each satisfied by $\sigma=I_{h h}$, so that the indicatrix lies on each of them. In particular

$$
D_{\mathrm{a}}^{\prime}(\sigma) \equiv \sigma \cdot \psi^{\prime} \cdot \sigma
$$


where $\psi^{\prime}$ is

$$
2 \psi^{\prime}=\sum_{r s t u}\left(a_{r s} a_{t u}-a_{r t} a_{s u}\right) x_{r s}^{\prime} x_{t \imath \iota}^{\prime}
$$

13. INTERSECTION OF CONSECUTIVE NORMAL $\boldsymbol{P}_{n-m}$ 'S

Consider any point in the normal $P_{n-m}$. It is given by the formula

$$
y=x+\varrho \sigma
$$

in which $\sigma$ has its previous meaning of any unit vector in the normal $\boldsymbol{P}_{n-m}$ and $\varrho$ is a parameter. A point in the normal $P_{n-m}$ for a neighboring point of the variety is

$$
z=x+\delta x+(\varrho+\delta \varrho)(\sigma+\delta \sigma)
$$

and if these two $P_{n-m}$ 's intersect, then for their points of intersection

$$
\delta x+\varrho \delta \sigma+\sigma \delta \rho=0
$$

neglecting terms of higher order. When all differentials are written as derivatives with respect to $s$, this becomes

$$
\sum_{r} x_{r} \lambda^{r}+\varrho \sum_{r} \sigma_{r} \lambda^{r}+\sigma \sum_{r} \varrho_{r} \lambda^{r}=0
$$

and we can eliminate the last term by dot-multiplying in $x_{s}$, thus getting

$$
\sum_{r} a_{s r} \lambda^{r}+\varrho \sum_{r} \sigma_{r} \cdot x_{s} \lambda^{r}=0
$$

And since $\sigma$ is perpendicular to $x_{s}$ we can write this in the form

$$
\sum_{r} a_{s r} \lambda^{r}+\varrho \sum_{r} \sigma \cdot x_{s r} \lambda^{r}=0
$$

which is simply another way of writing (20), this $\varrho$ being the reciprocal of the one there used. Hence if any $\sigma$ is given, equations (20) and (21) determine the directions in the variety for which consecutive normal $\boldsymbol{P}_{\boldsymbol{n}-\boldsymbol{m}}$ 's intersect in points of the line determined by $\sigma$. Equation (21) shows that there are $m$ such points of intersection along the line determined by any direction $\sigma$.

If we set $\tau=\rho \sigma$ the equation (21) becomes

$$
\left|\tau \cdot x_{r s}-a_{r s}\right|=0
$$


and if in this we substitute

$$
\tau=m h^{\prime}+r
$$

the above reduces to

$$
\left|r \cdot x_{r s}\right|=0
$$

which may be written in terms of the invariants of the net as

$$
\left|r \cdot I_{i j}\right|=0
$$

The geometric interpretation of this is as follows. In case the space determined by the $I_{i j}$ is the total normal $P_{n-m}$, the equation (46) represents a hypercone, so that the locus of the points of intersection of consecutive normal $\boldsymbol{P}_{\boldsymbol{n}-\boldsymbol{m}}$ 's is a hypercone of order $m$, lying in the normal $\boldsymbol{P}_{\boldsymbol{n}-\boldsymbol{m}}$ and having its vertex at the point $x+m h^{\prime}$. This point is the point diametrically opposite the variety point $P$, on the hypersphere which is the inverse of the hyperplane (41) with respect to the unit hypersphere with $P$ as center. In the more general case the locus is still defined by (46) and we have:

Adjacent normal $\boldsymbol{P}_{n-m}$ 's intersect in a $\boldsymbol{P}_{n-2 m}$ which is perpendicular to the space defined by the $I_{i j}$ and which contains in it an element of the hypercone (46).

DARTMOUTH COLLEGE,

HANOVER, N. H. 